\title{
THE COMPARISON OF DRAINAGE NETWORK EXTRACTION BETWEEN SQUARE AND HEXAGONAL GRID-BASED DEM
}

\author{
Lu Wang ${ }^{1}$, Tinghua $\mathrm{Ai}^{1 *}$ \\ ${ }^{1}$ School of Resource and Environmental Sciences, Wuhan University, Wuhan, China - (yulu_wang, tinghuaai)@whu.edu.cn
}

Commission IV, WG IV/8

KEY WORDS: Hexagonal grid-based DEM, Square grid-based DEM, D6 algorithm, Drainage networks extraction

\begin{abstract}
:
The automatic extraction of valleys or ridges from DEM is a long term topic in the GIS and hydrology fields and a number of algorithms have been developed. The quality of drainage networks extraction depends on many impacts such as data source, DEM resolution and extraction algorithms. However, little consideration has been paid to the influence of different tessellation of gridbased DEM construction in terrain surface representation. Actually, hexagonal grid has been proved to be advantageous over square grid due to its consistent connectivity, isotropy of local neighbourhoods, higher symmetry, visual advantage, and so on. This study tries to explore the impact of different tessellation scheme for grid-based DEM on the accuracy of terrain representation reflected by the results of drainage networks extraction. The contour line data model is applied to grid-based DEM generation. Then, by analogy with traditional D8 algorithm, the D6 algorithm is introduced to extract drainage networks by calculating flow direction of each gird with the steepest slope neighbour criteria. From the comparison between D8 algorithm and D6 algorithm, we conclude that hexagonal grid-based DEM has a superior capability in maintaining the detailed shape and the characteristics of extracted drainage networks in coarser resolution.
\end{abstract}

\section{INTRUDUCTION}

Grid-based DEM is the simulation and expression of real land surface space by the elevation of the discrete sampling points. It has been an important data source to automatic extraction of drainage networks due to its characteristics of simple data structure, computational efficiency, and so on.

The flow paths in DEMs are generated based on elevation differences among grid cells and their downslope neighbouring cells. So, the extraction of drainage channels relies not on the isolated elevation sampling points but on the spatial relationship between these points (Vaze et al., 2010). However, traditional researches have regarded data source, resolution and interpolation method as main factors to measure the extraction accuracy (Sousa et al., 2017; Ariza-Villaverde et al., 2015; Woodrow et al., 2016). They ignore the impact of different shape of regular grid on terrain surface representation of DEM, which plays a role in results of streaming extraction.

Actually, there are three regular tessellation schemes for space: triangles, squares and hexagons, as presented in Figure 1. However, triangles are rarely considered for tessellation model, because there is inherent orientation variability in model, making analysis and computation across grids more complex (Raposo, 2013). Square grid-based DEM has been a general data standard of DEM because square are suitable for computer processing, storage and calculation in the form of matrix. There is less researches for hexagonal grid-based DEM and the difference of grid-based DEM between squares and hexagons. It is the original intention of this study to explore the impact of different tessellation scheme for grid-based DEM on the results of drainage networks extraction.

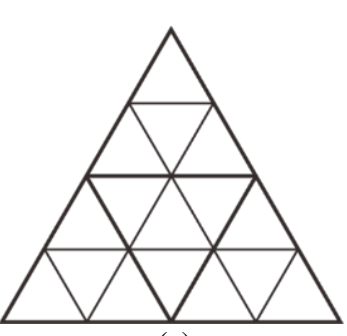

(a)

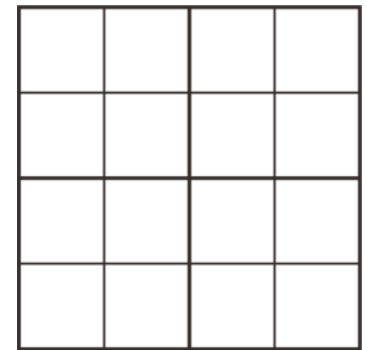

(b)

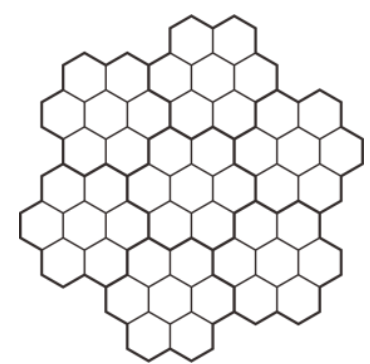

(c)

Figure 1. Three regular tessellation schemes for space: (a) triangles; (b) squares; (c) hexagons.

The hexagonal grids have been explored to image processing, game, cartography, ecology and other fields due to its theoretical advantages over square (Birch et al., 2007; Carr et al., 1992; Singh et al., 2015; Wang et al., 2018). Singh et al. (2015) has discussed the characteristics of hexagon applied in image processing. They conclude that hexagon grid is an alternative tessellation scheme besides the conventional square grid for sampling and representing images, and it is advantageous over square pixel because of its higher symmetry, higher sampling efficiency, equidistance, greater angular resolution, less aliasing effect, consistent connectivity. Based on 
the simpler nearest and less ambiguous neighbourhood (Figure 2) in a hexagonal grid, Birch et al. (2007) propose that the hexagonal grid should be preferred when it has advantages for the construction or representation of nearest neighbourhood, movement or connectivity in ecological models. What's more, hexagonal gird is closer to a circle, which makes the hexagonal tessellation model have a more compact structure (Hales, 2001). So, as the grid cell in raster data structure, the hexagon has the shorter perimeter compared with square and triangle, which help to improve the accuracy of raster data with same data volume, or reduce the data volume apparently under the same accuracy (Lin et al., 2001). However, a hexagon cannot be divided in smaller hexagons, which limits the application of hexagonal tessellation model to DEM. Thus, there are only a few studies on DEM based on hexagonal grids.

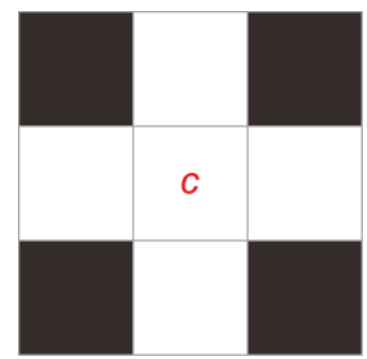

(a)

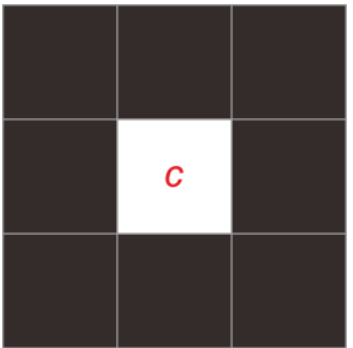

(c)

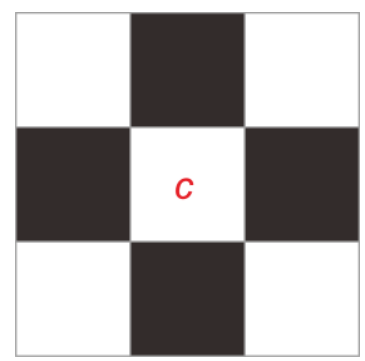

(b)

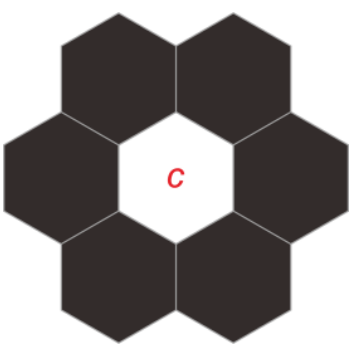

(d)
Figure 2 . The connectivity between grids in square and

hexagonal tessellation models. (a) 4-neighbourhood connectivity defined by corner contact of square tessellation model; (b) 4-neighbourhood connectivity defined by edge contact of square tessellation model. (c) 8-neighbourhood connectivity of square tessellation model; (d) 6-neighbourhood connectivity of hexagonal tessellation model.

Given the higher resolution with the same number of sampling points and the isotropy of neighbourhoods in hexagonal grids, de Sousa et al. (2006) prove that the hexagonal grid-based DEM has a superior capacity in maintaining the original flow directions. Based on this study, Wright et al. (2014) propose a Hierarchical Hexagonal Surface Model to store and process hexagonally sampled data. These two studies have demonstrated that hexagonal data sampling has advantages over rectangular grids for hydrological modelling from flow direction calculation. What's more, the hexagonal grid-based DEM in these studies is resampled by traditional square grid-based DEM, which fails to avoided the influence of elevation sampling by square. And its analysis results are specific to flow direction, which is only one of steps for drainage networks extraction.

In view of the above analysis, this study tries to establish hexagonal grid-based DEM and square grid-based DEM by contour line data respectively. Based on the generated DEMs, we explore the differences of drainage networks extracted by D6 algorithm and D8 algorithm.

\section{METHODOLOGY}

\subsection{Gird-based DEM generation}

Taking the data accessibility and the compatibility of two gridbased DEMs into consideration, the contour line data model is applied to grid-based DEM generation (Chen et al., 2013). And the TIN generation transformed from contour lines as an assistant process is utilized to simplify the interpolation and improve computer efficiency. The detailed generation procedure of grid-based DEM includes the following steps:

(1) Divide the study region into a set of basic grids (both square and hexagon), where the size of grid depends on the application aims of DEM. Based on the basic grids and the topological relationship between these grids, the respective tessellation model is established.

(2) According to the view of "point raster" (Tang et al., 2005), the elevation value of each gird is expressed by the value of central point. The elevation value of each central point is calculated by linear interpolation method within corresponding triangle of TIN.

The linear interpolation method based on triangle in TIN model is a special case of block interpolation algorithm. Set the geographical coordinates and elevation value of the interpolation point $\mathrm{P}$ as $\left(x_{p}, y_{p}\right)$ and $z_{p}$. The point is contained in triangle $\Delta V_{1} V_{2} V_{3}$ with the geographical coordinates $\left(x_{1}, y_{1}\right),\left(x_{2}, y_{2}\right),\left(x_{3}, y_{3}\right)$ and elevation $z_{1}, z_{2}, z_{3}$ of vertices $V_{1}, V_{2}, V_{3}$, as shown in Figure 3 .

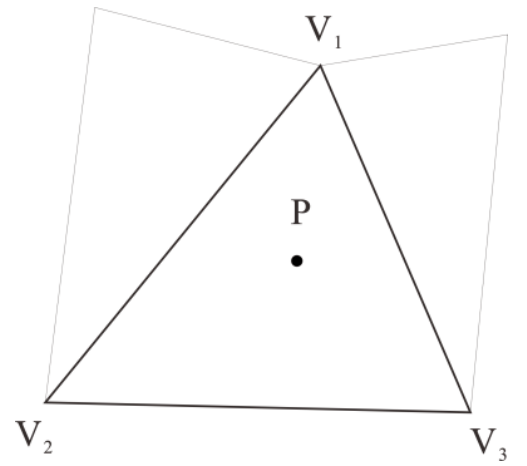

Figure 3. The schematic diagram of the linear interpolation method based on triangle.

The plane decided by triangle $\Delta V_{1} V_{2} V_{3}$ can be expressed as:

$$
\left|\begin{array}{llll}
x_{p} & y_{p} & z_{p} & 1 \\
x_{1} & y_{1} & z_{1} & 1 \\
x_{2} & y_{2} & z_{2} & 1 \\
x_{3} & y_{3} & z_{3} & 1
\end{array}\right|=0
$$

Then, the $z_{p}$ can be calculated by following formula:

$$
z_{p}=z_{1}-\frac{\left(x_{p}-x_{1}\right)\left(y_{21} z_{31}-y_{31} z_{21}\right)+\left(y_{p}-y_{1}\right)\left(z_{21} x_{31}-z_{31} x_{21}\right)}{x_{21} y_{31}-x_{31} y_{21}}
$$


where

$$
\begin{aligned}
& x_{21}=x_{2}-x_{1} ; x_{31}=x_{3}-x_{1} \\
& y_{21}=y_{2}-y_{1} ; y_{31}=y_{3}-y_{1} \\
& z_{21}=z_{2}-z_{1} ; z_{31}=z_{3}-z_{1}
\end{aligned}
$$

(3) Output the gird-based DEM data.

\subsection{The extraction of drainage networks}

The classical method of automatic drainage networks extraction is applied both for square and hexagonal grid-based DEMs. It includes depression filling, flow direction, flow accumulation calculation and accumulation threshold definition. The extraction conforms to the natural characteristic of overland runoff. That is to say, the streaming always run along the steepest direction of the slope which can be calculated by the elevation differences between neighbour grids in DEM structure So, the flow direction is the crucial step. With similar to D8 algorithm proposed by O' Callaghan et al. (1984), the D6 algorithm is introduced to define the neighbour with lowest elevation value as the flow direction for hexagonal grid-based DEM.

The progress of flow direction extraction based on D6 algorithm includes flow direction coding, sloping calculation of each neighbourhood and the flow direction defined by the max sloping value.

Based on the features of consistent connectivity and isotropy of hexagon, the D6 algorithm applied in lower resolution may preserve flow direction characteristics of higher resolution to a great extent (de Sousa et al., 2006). It suggests that the results of automatic extraction of valleys based on D6 algorithm between different resolutions may maintain consistency of shape characteristic.

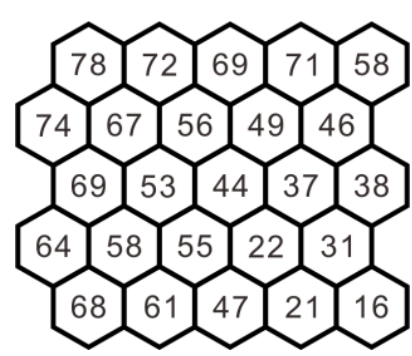

(a)

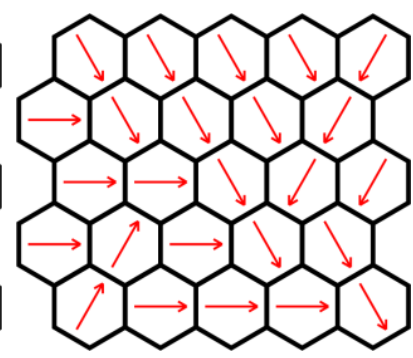

(b)

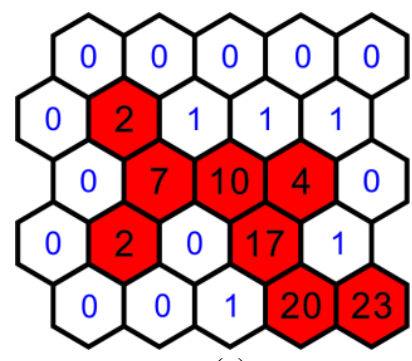

(c)

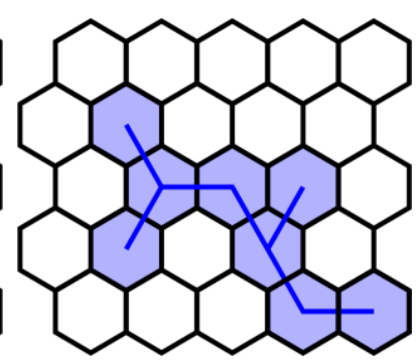

(d)
Figure 4. The schematic diagram of drainage networks extraction based on D6 algorithm: (a) the elevation data; (b) the estimated result of flow direction displayed by arrows; (c) the calculated result of flow accumulation; (d) the result of extraction with accumulation threshold of 2.

\section{EXPERIMENT AND DISCUSSION}

\subsection{The generation results of grid-based DEM}

We choose northwest of Deyang city, Sichuan Province, China as the study region. The contour lines with a contour interval of $10 \mathrm{~m}$ are utilized to generate DEM based on square grids and hexagonal grids. To ensure the same sampling density in tessellation model of hexagons and squares, same resolution is defined as the same area between a hexagon and a square with the side length radio of $\sqrt{3 \sqrt{3} / 2}$.

Based on the method mentioned in Section 2.1, we respectively establish different resolutions of hexagonal gird-based DEM and square gird-based DEM with corresponding horizontal grid widths in Table 1.

\begin{tabular}{|c|c|c|c|c|}
\hline ID & \multicolumn{2}{|c|}{ Horizontal grid width $(m)$} & \multicolumn{2}{c|}{ Number of grids } \\
\hline & $\begin{array}{c}\text { Square } \\
\text { DEM }\end{array}$ & $\begin{array}{c}\text { Hexagonal } \\
\text { DEM }\end{array}$ & $\begin{array}{c}\text { Square } \\
\text { DEM }\end{array}$ & $\begin{array}{c}\text { Hexagonal } \\
\text { DEM }\end{array}$ \\
& & & 146376 & 146183 \\
1 & 46.5 & 50 & 36601 & 36535 \\
2 & 93 & 100 & 16258 & 16293 \\
3 & 139.5 & 150 & 9150 & 9131 \\
4 & 186 & 200 & 5846 & 5848 \\
5 & 232.5 & 250 & 4046 & 4055 \\
6 & 279 & 300 & 2990 & 2983 \\
7 & 325.5 & 350 & 2291 & 2281 \\
8 & 372 & 400 &
\end{tabular}

Table 1. The horizontal grid width and the number of grids for established hexagonal gird-based DEM and square gird-based DEM with same resolution.

(Note: In following paper, the resolution value is based on the horizontal grid width of hexagon.)

As shown in Figure 5(a) and (c), from the perspective of visualization, the generation results of higher resolution between these two gird-based DEM are similar. But in the lower resolution results, the regional outline and the lines between different elevation values are more clear and smooth represented by hexagonal gird-based DEM, as in Figure 5 (b) and (d). 


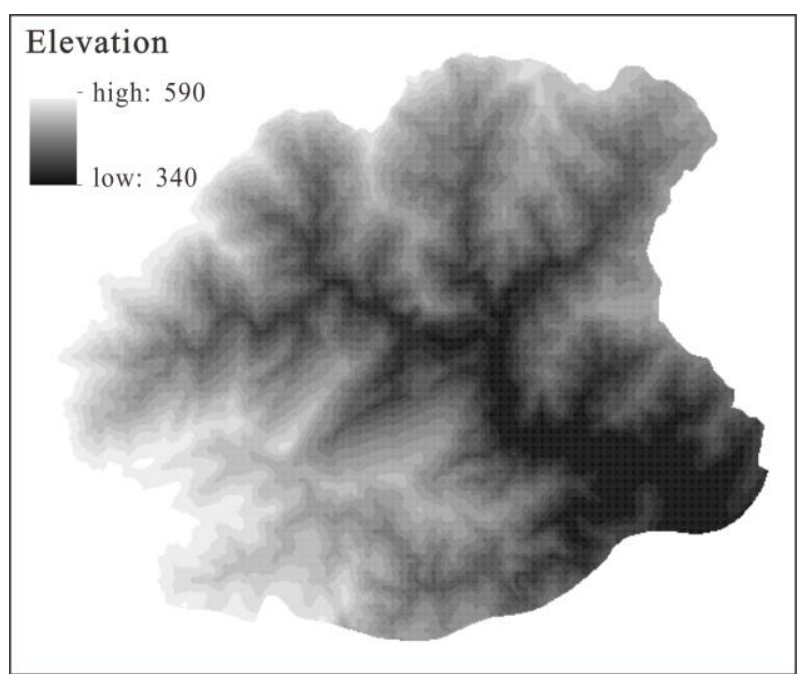

(a)

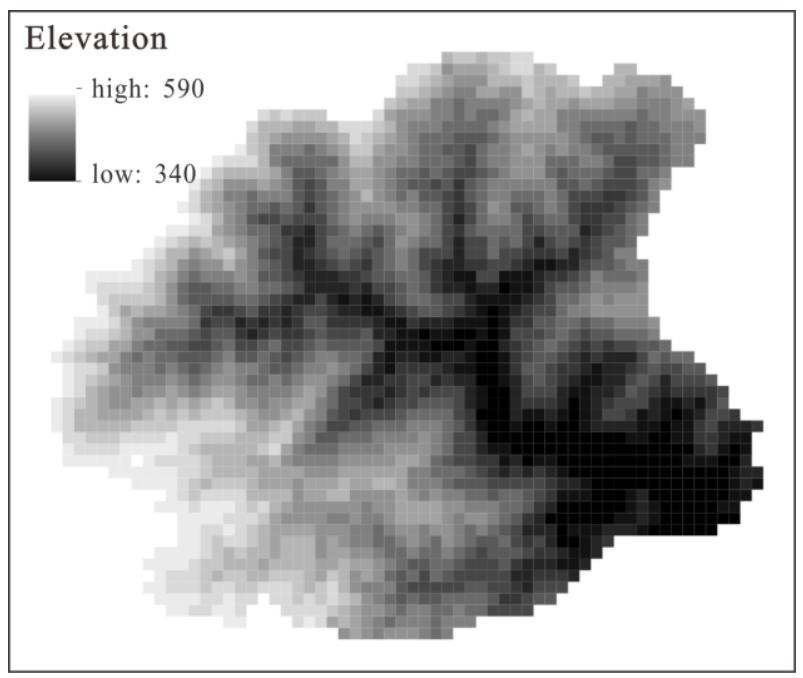

(b)

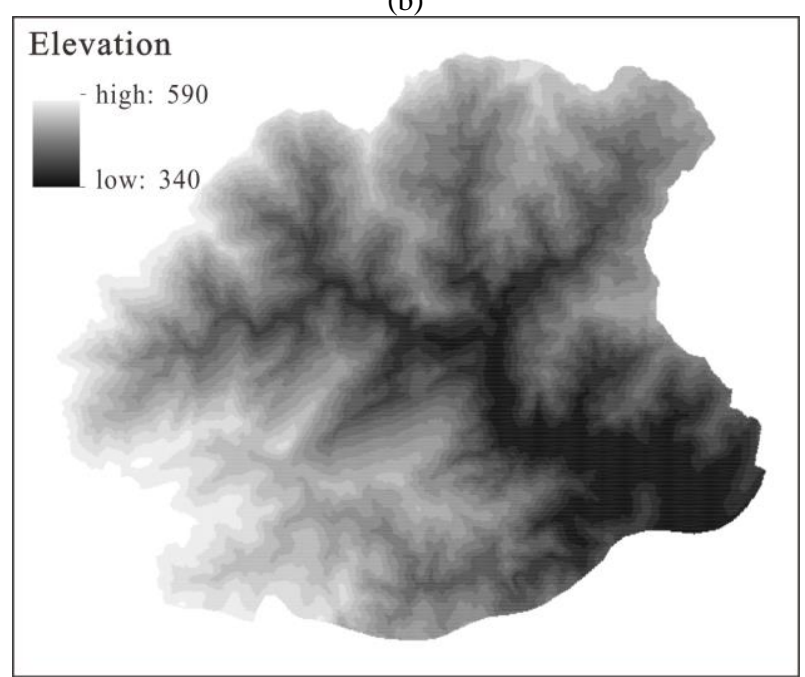

(c)

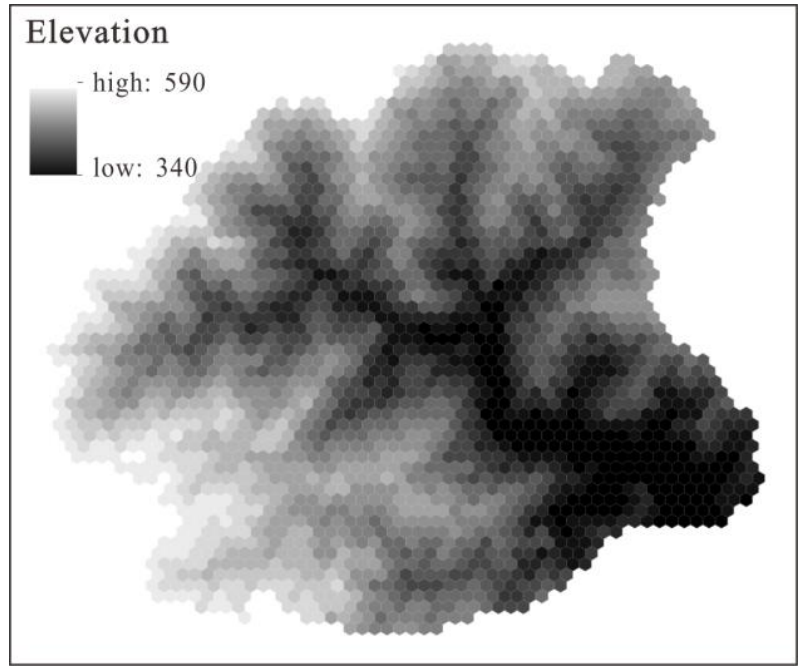

(d)

Figure 5. The comparison of generation results between

hexagonal and square grid-based DEMs at $50 \mathrm{~m}$ and $400 \mathrm{~m}$ resolution:(a) square grid-based DEM with 50m resolution; (b) square grid-based DEM with 400m resolution; (c) hexagonal grid-based DEM with 50m resolution; (d) hexagonal grid-based DEM with $400 \mathrm{~m}$ resolution.

\subsection{The results of drainage networks extraction}

Based on the generated grid-based DEM both of hexagons and squares at different resolutions, the drainage networks are extracted with flow accumulation threshold of 25 by D6 algorithm and D8 algorithm respectively. With the decrease of resolution, the number of streaming extracted from these two grid-based DEMs becomes less, the branches of the river networks are lost and the shape feature of the river is gradually simplified.

To measure the loss rate of extracted drainage networks characteristics in coarser resolution, we choose the extracted drainage networks of $50 \mathrm{~m}$ resolution as the reference data. As demonstrated in Figure 6, comparing the extraction results between D6 algorithm and D8 algorithm in 50m resolution, the differences are mostly shown in lower level branches, which flow into the trunk at different angles due to the difference angle resolutions between hexagon and square. And the extracted backbones rivers are similar in these two results. However, comparing the extraction results between D6 algorithm and D8 algorithm in 400m resolution, the rivers are smoother extracted by hexagonal grid-based DEM. The backbones rivers extracted by D8 algorithm are simplified at a larger degree, especially for the longitudinal rivers. 


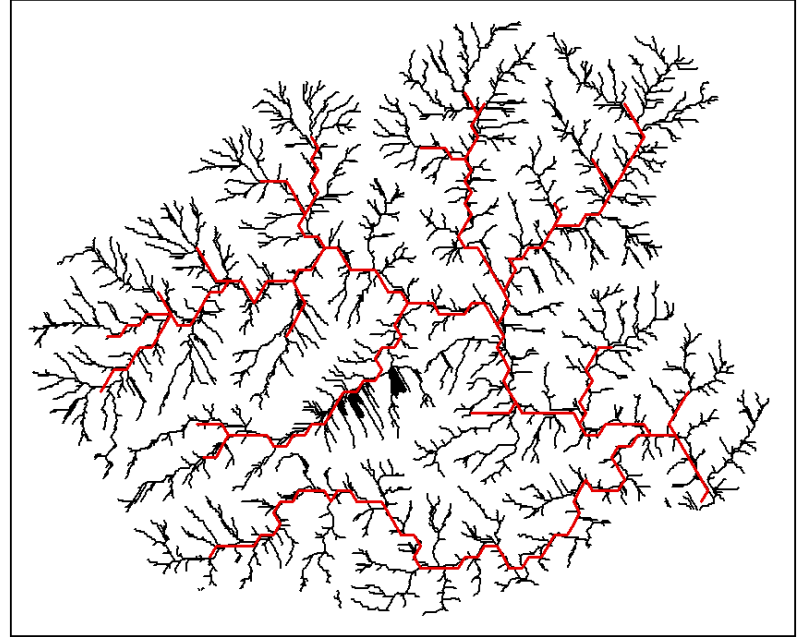

(a)

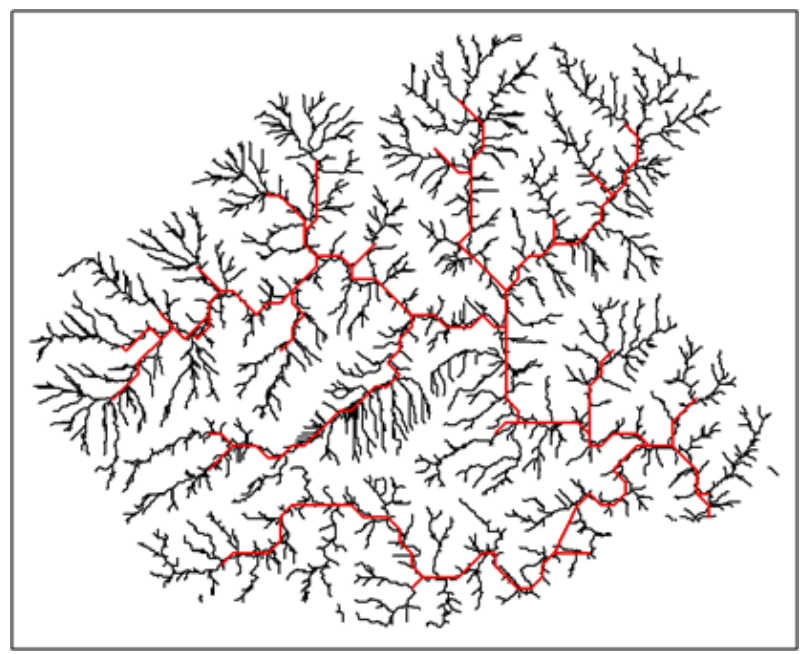

(b)

Figure 6. The comparison of drainage networks extraction results between hexagonal and square grid-based DEMs at $50 \mathrm{~m}$ and $400 \mathrm{~m}$ resolution: the black lines are extracted results at $50 \mathrm{~m}$ resolution, the red lines are extracted results at $400 \mathrm{~m}$ resolution. (a) the results based on D6 algorithm; (b) the results based on D8 algorithm.

\section{CONCLUSIONS}

This paper demonstrates that the tessellation scheme of gridbased DEM also plays a role in terrain representation and results of automatic drainage networks extraction. With the novel relationship between neighbourhood grids, the hexagonal grid-based DEM has a superior capability in maintaining the characteristics of extracted drainage networks between different resolutions. It also provides an innovative model for analysis of other DEM-derived topographic features.

However, this paper only focuses on the simplest model of drainage networks extraction to compare the difference in two grid-based DEMs. So, the future study will pay attention to the following aspects: (1) the impact of tessellation scheme on terrain factors extraction. Because topography can be used to develop more physically realistic models of hydrological; (2) the suitable flow direction algorithm for flat area in hexagonal grid-based DEM.

\section{REFERENCES}

Ariza-Villaverde, A. B., Jiménez-Hornero, F. J., \& de Ravé, E. G., 2015. Influence of DEM resolution on drainage network extraction: A multifractal analysis. Geomorphology, 241, 243254.

Birch, C. P., Oom, S. P., \& Beecham, J. A., 2007. Rectangular and hexagonal grids used for observation, experiment and simulation in ecology. Ecological modelling, 206(3-4), 347-359.

Carr, D. B., Olsen, A. R., \& White, D., 1992. Hexagon mosaic maps for display of univariate and bivariate geographical data. Cartography and Geographic Information Systems, 19(4), 228236.

Chen, C., \& Li, Y., 2013. An orthogonal least-square-based method for DEM generalization. International journal of geographical information science, 27(1), 154-167.

de Sousa, L., Nery, F., Sousa, R., \& Matos, J., 2006. Assessing the accuracy of hexagonal versus square tilled grids in preserving DEM surface flow directions. In: Proceedings of the 7th International Symposium on Spatial Accuracy Assessment in Natural Resources and Environmental Sciences, pp. 191-200.

Hales, T. C., 2001. The honeycomb conjecture. Discrete \& Computational Geometry, 25(1), 1-22.

Lin, H., \& Peng, C. H., 2001. The Role of Raster Pixel Size and Shape in Geographic Information System. Remote Sensing Information, 1(1), 21-23.

O'Callaghan, J. F., \& Mark, D. M., 1984. The extraction of drainage networks from digital elevation data. Computer vision, graphics, and image processing, 28(3), 323-344.

Raposo, P., 2013. Scale-specific automated line simplification by vertex clustering on a hexagonal tessellation. Cartography \& Geographic Information Science, 40(5), 427-443.

Singh, I., \& Oberoi, A., 2015. Comparison between square pixel structure and hexagonal pixel structure in digital image processing. International Journal of Computer Science Trends and Technology, 3(1), 176-181.

Sousa T. M. I., Paz A. R., 2017. How to evaluate the quality of coarse-resolution DEM-derived drainage networks. Hydrological Processes, 31(13), 3379-3395.

Tang, G. A., Liu, X. J., Lü G. N., 2005. Principles and Methods of Digital Elevation Model and Geo-science Analysis. Science Press, Beijing, pp. 28-30.

Vaze, J., Teng, J., \& Spencer, G., 2010. Impact of DEM accuracy and resolution on topographic indices. Environmental Modelling \& Software, 25(10), 1086-1098.

Wang, J., \& Kwan, M. P., 2018. Hexagon-Based Adaptive Crystal Growth Voronoi Diagrams Based on Weighted Planes for Service Area Delimitation. ISPRS International Journal of Geo-Information, 7(7), 257.

Woodrow, K., Lindsay, J. B., \& Berg, A. A., 2016. Evaluating DEM conditioning techniques, elevation source data, and grid 
resolution for field-scale hydrological parameter extraction. Journal of Hydrology, 540(1), 1022-1029.

Wright, J. W., Moore, A. B., \& Leonard, G. H., 2014. Flow direction algorithms in a hierarchical hexagonal surface model. Journal of Spatial Science, 59(2), 333-346. 